\title{
G

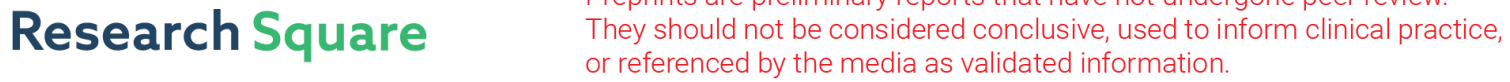 \\ Effects of Orthoptic Therapy in Children with Intermittent Exotropia After Surgery: Study Protocol for a Randomized Controlled Trial
}

\section{Meiping Xu}

Eye Hospital and School of Ophthalmolody and Optometry, Wenzhou Medical University https://orcid.org/0000-0002-8755-3091

\section{Fuhao Zheng}

Wenzhou Medical University Eye Hospital

\section{Tingting Peng}

Wenzhou Medical University Eye Hospital

\section{Chunxiao Wang}

Wenzhou Medical University Eye Hospital

Jiangtao Lou

Wenzhou Medical University Eye Hospital

Huanyun Yu

Wenzhou Medical University Eye Hospital

\section{Yuwen Wang}

Wenzhou Medical University Eye Hospital

Xinping Yu ( $\triangle$ yu-xinping@163.com )

Sun Yat-Sen University Zhongshan Ophthalmic Center

\section{Research Article}

Keywords: Intermittent exotropia, Orthoptic therapy, Suboptimal surgical outcome, Study protocol, Randomized controlled trial

Posted Date: December 2nd, 2021

DOI: https://doi.org/10.21203/rs.3.rs-877252/v1

License: (c) (1) This work is licensed under a Creative Commons Attribution 4.0 International License. Read Full License

Version of Record: A version of this preprint was published at Trials on April 11th, 2022. See the published version at https://doi.org/10.1186/s13063-022-06246-4. 


\section{Abstract \\ Background}

Intermittent exotropia (IXT) is the most common type of exotropia in China. Surgery is usually required to align the eye deviation to maintain or obtain better binocular visual function. However, there is a high rate of exodrift or recurrence in surgically treated patients. Orthoptic therapy is sometimes recommended for IXT patients after surgery. However, there is a lack of high-quality randomized controlled trials to prove that orthoptic therapy could be an effective supplement to surgical treatment for IXT patients. This study was designed to confirm such an application. This report describes the design and methodology of the Intermittent Exotropia Post-operative Treatment Clinical Trial, which is the first large-sample, blankcontrolled, randomized clinical trial.

\section{Methods}

A total of 136 IXT patients (aged 7 to 17 years) will be enrolled and assigned to the orthoptic therapy group or blank control group according to a simple randomization scheme. Patients in the orthoptic therapy group will receive at least 2 months of orthoptic therapy, such as anti-suppression, vergence, and accommodation training. Patients in the blank control group will receive only refractive correction. All enrolled patients will need regular follow-up observation until 2 years after surgery. The primary outcome will be the proportion of participants meeting suboptimal surgical outcomes in this 2-year follow-up, which is defined as (1) exodeviation of 10 prism diopters (PD) at distance or near using the simultaneous prism and cover test (SPCT) or (2) loss of 2 or more octaves of stereoacuity from baseline, at any masked follow-up visit examination. The secondary outcomes will be the exodeviation at distance and near using the simultaneous prism and alternate cover test (PACT), magnitude of fusional convergence, stereoacuity, and accommodation. Measurements will be taken at baseline and at the 6-, 12-, 18-, and 24month follow-ups.

\section{Discussion}

To the best of our knowledge, this will be the first prospective, randomized controlled study of binocular training in IXT patients after surgery. The aim of this work is to confirm the efficacy of orthoptic therapy in reducing the proportion of recurrence among IXT patients after surgery and improving binocular vision function.

\section{Trial registration:}

Chinese Clinical Trial Registry ChiCTR1900026891. Registered on 25 October 2019. 


\section{Background}

Intermittent exotropia (IXT) is the most common type of childhood-onset exotropia[1, 2]. At the same time, IXT is also the most common type of strabismus in China, and its incidence is approximately $3.26 \%[3]$. IXT patients usually present exodeviation when they are fatigued, distracted or looking at distance, and the alignment of the eye position is controlled when they are nervous or focused. With the progression of disease, there is poorer control of exodeviation, which leads to deterioration in binocular vision and negative psychosocial consequences[4,5]. In such cases, surgery is often the treatment of choice. A recent study also showed that IXT was the main contributor to increases in strabismus surgery in China[6]. However, whether surgery benefits long-term ocular alignment in IXT patients is unclear, as a high recurrence rate has been recently reported[7-10]. The most important factor determining surgical outcomes is the duration of follow-up. With extensions in follow-up time, the success rate of surgery shows a decreasing trend[11]. Kim et al. revealed that more than $50 \%$ of the total amount of exodrift occurred within the first post-operation year[12], and several other studies have reported that the main recurrence of exodeviation occurred within 2 years after surgery[13-15].

The main objective of orthoptic therapy for IXT patients is to remove suppression, correct abnormal retinal correspondence, improve sensory fusion and fusional reserve, and obtain better binocular vision. Previous studies have mainly focused on patients with small deviation IXT to obtain better fusional control[$[16,17]$. Using surgery combined with pre- or postsurgical, or both, nonsurgical treatments, including orthoptic training and occlusion therapy, has been advocated by different ophthalmologists in different countries[18-21], and such assessments have been based on clinical impressions rather than solid evidence. Therefore, a comprehensive, well-designed prospective study is urgently needed to determine the value of orthoptic therapy for IXT patients after surgery. The main purpose of this study was to confirm whether orthoptic therapy could improve the long-term stability of eye position in IXT patients after surgery. Therefore, we designed a randomized, controlled trial including two groups: an orthoptic therapy group and a control group to explore the efficacy of orthoptic training in IXT patients after surgery over a 2-year period.

\section{Methods}

\section{Study design}

This will be a prospective, parallel, simple randomized controlled trial. All procedures meet the tenets of the Declaration of Helsinki and were approved by the medical ethics committee of the Affiliated Eye Hospital of Wenzhou Medical University (2019-108-K-101). Written consent and verbal assent will be obtained from parents (or guardians) and participants, respectively. The study protocol (V2/2019.08.10) was registered in Chinese Clinical Trial Registry (ChiCTR1900026891), which is granted for public access, and is in accordance with the Standard Protocol Items: Recommendations for Clinical Interventional Trials (SPIRIT) guidelines[22]. The study will be performed in the Affiliated Eye Hospital of Wenzhou Medical University. The research flowchart is shown in Figure 1. 


\section{Participants and recruitment}

All participants will be recruited from an outpatient clinic. One of the investigators in this study will initially screen patients based on their eye position, age, address, and refractive status at one week after surgery. Then, an appointment will be made for a 1-month postoperative review with each patient who approximately meets the entry criteria. A well-trained investigator in the amblyopia and strabismus department will be responsible for the recruitment and management of participants. Patients meeting the eligibility criteria will be informed of the basic information related to and the requirements of the project. If patients and their parents (or guardians) are willing to participate, then they will be randomly enrolled after the initial baseline examination. All tests will be performed by the same senior physician who will be masked to the patient's randomization status. The inclusion and exclusion criteria are listed in Table 1.

\section{Tests performed at baseline and each follow-up visit}

After obtaining consent and assent, health-related quality of life (HRQOL) will first be assessed using the Intermittent Exotropia Questionnaire (IXTQ)[23]; these will be completed by each participant and their guardians in separate and quiet rooms. Then, each participant will undergo comprehensive ophthalmic examinations, including best corrected visual acuity (BCVA), subjective refraction, slitlamp biomicroscopy, and fundus examination. Then, strabismic and binocular visual examinations will be performed, including stereopsis, the amplitude of fusional vergence at far and near, monocular and binocular accommodative amplitude, monocular and binocular accommodative facility, near point of convergence, the prism and alternate cover test (PACT) and simultaneous prism and cover test (SPCT). The principal inspection sequence is first binocular then monocular without breaking fusion. If this principle is broken, for example, the monocular items are performed first, then before testing the binocular items, at least 15 minutes of rest for steady binocular fusion will be provided. The present study included a baseline visit and 6-, 12-, 18-, and 24-month follow-up visits. Each examination is completed on the same day. The 4 follow-up visits will fall into a \pm 2 -week range. The HRQOL was designed to be completed at the first examination time and at the last examination time. To ensure the quality and repeatability of data acquisition, all procedures will be performed by two experienced clinicians ( $M X$ and YW), and the procedures have been unified and standardized. The standard process regarding the important test items is as follows:

\section{Health-related quality of life questionnaire}

We will use the Intermittent Exotropia Questionnaire (IXTQ)[23] to assess the participants' and their guardians' health-related quality of life (HRQOL). The IXTQ consists of 3 components:

1. Child questionnaire: This will be used to assess the feelings of the child about his or her eye condition. Because the children enrolled in our study will be over 7 years old and have been attending primary school, they already have a certain level of understanding. We uniformly use the child questionnaire as the version for children aged 8 years and older, which consists of 12 items and a five-level response scale (never, almost never, sometimes, often, and almost always) and is 
self-administered as far as possible. Of course, if the child cannot understand the question by her or himself, there will be staff nearby to provide oral guidance and help.

2. Parent proxy questionnaire: This will be used to assess how parents think their child's eye condition affects their child. The questionnaire consists of 12 items, and a 5-level response scale, similar to the child questionnaire, is used.

3. Parental questionnaire: This will be used to assess the parents' feelings about their child's eye condition. This component of the questionnaire consists of 17 items and the 5-level response scale.

All questionnaires about the parents are completed by the parents themselves; if there is a part that they do not understand, the assistant staff can help to explain it. Parents and children are separated into two different rooms during the completion of the questionnaire to avoid interfering with each other.

\section{Exodeviation control at distance and near}

We will assess the exodeviation control at each follow-up period using the Office Control Score[24]. This assessment will be conducted before all other examinations. The investigator looks at the participant's eyes when he or she focuses at an accommodative target at distance $(6 \mathrm{~m})$ or near $(40 \mathrm{~cm})$ and determines a score based on the following scoring principle:

5 is constant exotropia

4 is exotropia $>50 \%$ of the 30 -second period before dissociation

3 is exotropia $<50 \%$ of the 30 -second period before dissociation

2 is no exotropia unless dissociated; recovers in $>5$ seconds

1 is no exotropia unless dissociated; recovers in $1-5$ seconds

0 is no exotropia unless dissociated; recovers in $<1$ second (phoria).

\section{Stereoacuity testing}

Near and distance stereoacuity will be assessed using the Titmus circles test and TNO at $40 \mathrm{~cm}$ and the Distance Randot Stereotest at $3 \mathrm{~m}$, respectively. Stereoacuity will be assessed with the participant's refractive correction. The Titmus circles measure from 40 to 800 seconds of arc (arcsec). The TNO test (Laméris Ootech B.V., Nieuwegein, the Netherlands) ranges from 15 to 480 arcsec. The Distance Randot Stereotest (DRS, American Stereo Optical Company U.S.A.) ranges from 63 to 400 arcsec. Testing starts with the largest disparity, and the inability to correctly identify the target with the largest disparity will be recorded as nil stereo. Nil stereoacuity will be assigned the next highest log level (near 1600 seconds of arc for Titmus; 960 seconds of arc for TNO; distance 800 seconds of arc for DRS) for the purpose of data analysis.

\section{Sensory fusion}


The Worth 4 Dot test will be conducted at a 40-cm and 5-m fixation to assess near and far sensory fusion, respectively. The participants will wear red-and-green spectacles and view the Worth 4 Dot flashlight at near and at distance. If participants report 2 red or 3 green lights, it will be taken to represent "suppression" of the left eye or right eye. If they report 4 lights at distance or at near, it means they have "normal" peripheral or central fusion. If 5 lights are reported, it will represent "diplopia".

\section{Fusional vergence}

The amplitude of fusional vergence will be assessed by a 1 to 40 PD prism bar for both near and far fixation. Each participant will fixate on a single letter (20/40 Snellen level) at $33 \mathrm{~cm}$ or $5 \mathrm{~m}$ and will be asked to report when the fixated letter appears to double (this will be recorded as the break point) as the prism strength gradually increases. Then, the prism power will be gradually decreased, and the point at which the patient regains single vision will be recorded as the recovery point. Participants will be reminded to keep the target single as long as possible. Both the divergence (BI) and convergence (BO) of the break point and recovery point are recorded at near and at distance. If the subject reports suppression, which will be assessed by the Worth 4 Dot test, then this part will be omitted and recorded as $0 / 0$. For subjects who still retain fusion at $45 \mathrm{PD}$, the break point will be recorded as $45 \mathrm{PD}$, and the recovery point will be recorded as "-" and excluded from the recovery analysis. The testing order for fusional vergence is distance first and then near, divergence first and then convergence. The PD values of break points are defined as the amplitude of the vergence, which is defined as convergence reserve in our study.

\section{Near point of convergence}

For this test, participants will fixate at a single Snellen 6/12 letter as an accommodation target at $40 \mathrm{~cm}$ in front of their eyes. The investigator will move the target closer to the subject and observe the subject's eyes until he or she reports diplopia or one eye drifts outward. This point will be recorded, and the distance from the point to the canthus or the plane of the subject's glasses will be measured.

\section{Accommodative function}

The accommodative function we measure here will include accommodative amplitude and facility. We will separately measure binocular and monocular function in both examinations. Generally, we will measure accommodative facility first and then amplitude, and we will measure binocular function first then monocular function. When measuring binocular function, we will always observe the subjects' eye position and remind them to maintain binocular single vision. The test will be stopped if the patient has diplopia or appears to have an outward deviation.

We will use standard test methods ( $\pm 2.00 \mathrm{D}$ flipper lenses) to test this facility. The subjects will be asked to read one line above the best visual acuity at $40 \mathrm{~cm}$ with their corrected lenses. Then, the $+2.00 \mathrm{D}$ lenses will be placed in front of the subjects' eyes, and when the letters are reported to be clear, the flipper $(-2.00 \mathrm{D}$ lens $)$ will be quickly flipped in front of the eyes; 1 cycle is achieved when clear is reported again. 
This will be continued while alternating sides of the flipper lenses for 1 minute, and the cycles achieved will be recorded.

We will use "Minus lens" to test the amplitude. A near target will be set at $40 \mathrm{~cm}$, minus lenses will be gradually added in front of the subjects' eyes until they report sustained blur. The summation of the number of minus lenses and 2.50 D for working distance will provide the total accommodative amplitude.

\section{Ocular alignment testing}

Ocular alignment will be assessed by SPCT and PACT both at near $(33 \mathrm{~cm})$ and at distance $(5 \mathrm{~m})$. The deviation will be recorded as constant or intermittent if a manifest exodeviation is present in at least 3 cover and uncover tests. The amount of manifest exodeviations will be recorded by the values of SPCT, and the total amount of exodeviations will be recorded by the measurement of PACT. If no deviation is present at any time, it will be recorded as "no deviation".

\section{Intervention}

After a subject completes all of the examinations, the checklist is handed to the research assistant. Subjects will be randomly divided into Group A (orthoptic therapy group) and Group B (control group) based on the order provided in the random table.

\section{Intervention for the orthoptic therapy group}

All participants in the orthoptic therapy group will receive at least 8 weeks of hospital-based orthoptic therapy combined with home reinforcement administered by an orthoptic therapist based on their baseline binocular visual status. The therapy protocol in the present study is based on participants' binocular vision status and modified on the basis of the Convergence Insufficiency Treatment Trial randomized clinical trial ${ }^{24,25}$. Hospital-based training will mainly include methods that are not easy to master, the operation is more complex, and the training equipment is not suitable for home use. Furthermore, some participants with poor visual function will need to complete this training under the guidance of a therapist. To enhance the treatment confidence of participants and parents, the therapeutic procedures will be arranged from easiest to most difficult. At the same time, therapists will often verbally motivate the participants to perform the more difficult tasks.

The participants will receive hospital-based therapy 1-2 times per week, 60 minutes each time, combined with practice at home for 15-30 minutes, 5 days per week. Therapists will also instruct parents to complete the training log and maintain communication through WeChat in an attempt to enhance compliance and improve the therapeutic effect. The overall treatment program will consist of 3 phases. In each phase there are a number of subcategories. The detailed therapeutic program is summarized in Table 2. The participants in our study will have normal binocular visual acuity and basic binocular visual function, which may be different from other patients with abnormal visual function. Each procedure has a designated endpoint that should be obtained before moving on to the next level or phase. 
Before the start of each training, some simple binocular function tests will be performed, and the training program will be adjusted based on those test results. The patients can stop training in the hospital and maintain home training ( 15 minutes each day) to maintain efficiency if the test results showed that performance had reached or even exceeded the normal level. However, if a decrease in the test results is observed during the follow-up, the appropriate therapeutic treatment will be reinitiated. For the results and purpose of our study, parents are required to record the training in detail, including time, frequency, experience, etc.

\section{Control group therapy}

For the participants in the control group, all binocular visual function tests will also be completely performed at each follow-up visit. If refractive status changes, appropriate prescription will be provided and used in a pair of new glasses.

If only binocular visual function deterioration occurs, it is proposed that they continue to be observed; if accompanied by a small angle of exodeviation but not meeting the criteria of suboptimal surgical outcome or if participants ask for visual function therapy, they will have deviated from the study protocol and will be considered to be out of group. If eye position regression is obvious and the definition of recurrence is reached, he or she will be classified as having a suboptimal surgical outcome. They will be offered a reoperation to align the eye position or start orthoptic therapy during the study.

\section{Outcome measurements}

Before the beginning of the study, we demonstrated the feasibility of the project, the reliability of the research methods and the evaluation of the research results. Stereopsis will be checked by the assistant medical staff of the department, and the other binocular vision items and the amount of exodeviation will all be checked by the same senior doctor.

\section{Primary outcome}

The primary outcomes in our study will be the proportion of participants meeting suboptimal surgical outcomes at any masked follow-up visit examination within 2 years of surgery; these suboptimal outcomes are defined as (1) exodeviation of $\geq 10$ PD at distance or at near using SPCT or (2) loss of 2 octaves or more of stereoacuity from baseline.

\section{Secondary outcomes}

The secondary outcomes in our study include the following items: the exodeviation at near and distance tested by PACT, fusional convergence amplitude, stereoacuity, accommodative amplitude and facility.

\section{Sample size}

A total sample size of 136 subjects (68 per group) are required for this study based on the following considerations: the proportion of participants meeting suboptimal surgical outcomes in the PEDIG study 
was $36.5 \%$ in 24 months[21]. Edwin et al. ${ }^{22}$ confirmed that the successful treatment rate among IXT patients was $83.3 \%(25 / 30)$ in the surgery combined with nonsurgical binocular therapy group. Statistical power was set at $80 \%$ with a type 1 error probability (a) of 0.05 based on a 2-tailed t test. The theoretical sample size was 61 , with a $1: 1$ sample ratio. To allow for a maximum drop-out rate of $10 \%$ during the 2year follow-up, the sample size for each group was estimated at 68.

\section{Randomization}

A biostatistician will generate a simple randomized number list at a ratio of 1:1 using SPSS software version 20.0 (IBM Corp., Armonk, NY, USA). Randomization will be conducted on the day that all the enrolment tests are completed. After each subject is enrolled and has completed all of the examinations, he or she will be invited to the principal investigator. The investigator will group the subjects in accordance with the order of the numbers in the randomization table. If the participant is assigned to the blank control group, he or she will be informed to follow the routine clinical follow-up time, that is, 6 months, 12 months, 18 months and 24 months. In addition, an appointment will be made for the next follow-up. If the participant is assigned to the orthoptic therapy group, he or she will be given the detailed therapeutic schedule. If the patient is not satisfied with the group assignment after randomization and does not want to participate in the follow-up study, that patient will be dropped from the study. All procedures will be performed by two investigators ( $M X$ and $\mathrm{YW}$ ), and they will be blinded to the interventions.

\section{Data management and monitoring}

To ensure the successful conduct of research and data accuracy, we will conduct detailed training for all project team members before the study starts, and training will include the collection of patient information, examination process and methods, data recording and input, etc. Each patient will have complete case report forms (CRFs), where researchers will record the data. If a mistake is made while recording the data, any changes to a CRF will need to be signed and dated. Because of the possibility of exodrift over the prolonged time after surgery, if patients miss one or two follow-up visits, we will use the last obtained follow-up data instead. Data administrators will enter data into EpiData 3.1. It will be entered and checked by two full-time staff (TP and CW) from our clinical research centre, who will also be blinded to the interventions. The Clinical Trial Centre Office of the Eye Hospital of Wenzhou Medical University is responsible for data monitoring and overseeing the whole study. The original data, including private information, will be kept in the Data Monitoring Committee, which as an important department in Clinical Trial Centre Office. If there is any reasonable request and an agreement with the principal investigator is obtained, the data will be accessible through the research centre. The principal investigator will regularly report the research progress to the co-workers on the study, and the final results will be presented in the form of published articles. Authorship will be determined based on an individual's contribution to the study and in accordance with authorship eligibility guidelines. The ideas and scientificity underlying the research are very important. 


\section{Safety monitoring}

To protect the rights and interests of patients, all participants and their guardians will be informed of the potential benefits and possible risks of the trial before they enrol. The whole process will be carried out in a separate and quiet room by a trained professional assistant (JL). After confirming that the patient and guardian understand the relevant content, we will ask both the patient and his or her guardian to sign an informed consent form. If any one of these individuals do not sign the informed consent form, they will not be admitted to the study.

Because the orthoptic therapy being used in our study is a form of functional training, which is a noninvasive operation, the risk of harm to patients is very small. However, we will still inform all individuals of the training process in detail and will give them the researcher's telephone or WeChat to communicate at any time. All participants will be permitted to withdraw or terminate the study for any reason during the study, and the reasons will be recorded. Discontinuation or irregular orthoptic therapy will not be a reason for withdrawal from the study, and participants could complete follow-up visits provided if they are willing.

\section{Statistical analysis}

Statistical analysis will be performed using SPSS software version 20.0 (IBM Corp., Armonk, NY, USA). The parameters to be calculated are the means \pm standard deviations for the continuous variables and the rates (proportions) for the categorical variables.

For the primary analysis, the cumulative proportion of participants meeting the criteria for suboptimal surgical outcomes by 2 years will be compared between the 2 groups using the chi-square test. An intergroup difference and a corresponding $95 \%$ confidence interval (Cl) will also be calculated. Each of the 2 individual components of the specified suboptimal surgical outcome criteria will be assessed as a secondary outcome using the chi-square test.

For all participants who complete the 2-year visit, and after the data entry is completed, the statistician will separate the two groups of data based on the random table. Changes in exodeviation measured by PACT, stereoacuity, accommodative amplitude and facility, and fusional convergence amplitude between baseline and the 2-year time points will be compared between the two groups in linear regression models that will be adjusted for the corresponding baseline values. Additionally, considering that binocular visual function is different in different age, the final data may be divided into two subgroups according to different ages.

\section{Discussion}

The success rate following IXT surgery has been quite variable across studies, which can be attributed to differences in examination methods, criteria for surgical failure, and length of follow-up [8, 21, 25, 26]. It was confirmed that there is a high rate of exodrift or recurrence after surgery in IXT patients. A previous 
study has indicated that orthoptic therapy is effective in the treatment of convergence insufficiency IXT with a maximum deviation of $25 \mathrm{PD}[17,18]$. It has also been applied as a component of a postoperative combination therapy[21] but in a retrospective small sample study. Therefore, there are still many related unanswered questions, such as which patients will respond to this therapy, how long and at what frequency does orthoptic therapy have to be performed for maximal efficacy, what is the endpoint of treatment and what is the long-term stability of these results. It is our expectation to answer several of these questions with our present study.

One-month post-surgery is an important follow-up time. Discomfort caused by strabismus operations, such as red and swollen eyes, has basically disappeared. At that time, patients can rotate the eyeball freely and complete the relevant visual function examination. Therefore, the time point chosen for this study was 1 month after the operation, and the patients in the training group began training 1 month after the operation. In addition, our inclusion and exclusion criteria are relatively strict and may exclude many patients in clinical practice, such as those with esophoria, combined with diplopia and vertical deviation, amblyopia and ansometropia. In this manner, the confounding factors in enrolled patients are minimized, and the results will represent a powerful conclusion regarding the internal validity of this study. We have a detailed and clear therapeutic program for IXT patients in the training group, including the establishment of therapeutic stages and therapeutic endpoints for each stage. This hospital-based therapy for one patient requires one orthoptic therapist to assist the training. Home-based training requires recording training logs, and computerized training would allow the real-time detection of training times and intensity. It would be helpful for us to fully capture all aspects of the therapeutic situation of each patient and analyse correlations between therapy cooperation and the actual effects of orthoptic therapy.

There are several limitations in our present study. First, the inclusion and exclusion criteria for this study are relatively strict and may not fully reflect the effect of orthoptic therapy on the exodrift of all postoperative IXT patients. Second, this study uses only simple randomization, not a dynamic randomization technique based on the patient's surgical methods, age, diopter and other data, considering the weekly operability and our large sample. These effects will be taken into account during analysis of the later results of the study.

In summary, our study will be the first large-sample, prospective randomized controlled study to confirm the effect of surgery combined with orthoptic therapy in IXT patients. We hope that our research can provide strong evidence to guide clinical application.

\section{Trial Status}

The trial protocol presented here has obtained approval from the Affiliated Eye Hospital of Wenzhou Medical University Review Board (2019-108-K-101), and is aligned with protocol version 2, approved on 10 August 2019. The recruitment started on 20 August 2019 and completed on 17 August 2021. Considering the importance of standardized visual function examination and orthoptic training in our 
study, and hoping that our research can be extended and traced, we wrote this study protocol, even though recruitment has already completed.

\section{Declarations}

Acknowledgements We would like to thank all the staff and participants in this study.

Funding/Support General Scientific Research Projects of Zhejiang Provincial Education Department (Grant No. Y201942103) and Zhejiang Provincial Natural Science Foundation of China (Grant No. $\mathrm{LY} 19 \mathrm{H} 120004)$. The funding approved our study design and will monitor our progress during the research and review our conclusions after the trial completed.

Contributors $\mathrm{XY}$ conceptualized the study. MX designed the study and drafted the manuscript. YH helped to design the study. XY revised the manuscript. FZ, YW, HY and JL will perform this study. TP and CW are managing the data. $M X$ will perform the statistical analysis. $M X, H Y, X Y$ will analyse and interpret the data. All authors provided a final review and approved the manuscript before submission.

Ethics approval and consent to participate This trial was approved by the medical ethics committee of the Affiliated Eye Hospital of Wenzhou Medical University (2019-108-K-101). All participants and their guardians will be informed and asked to sign the consent form before they participate in the study.

Consent for publication All authors have read and agreed to the final version of the manuscript.

Competing interests, A computerized binocular vision therapy procedure was provided by Guangzhou Shijing Medical Software Co., Ltd. (Tel: +86 4008-777-511; Email: doboso@topeye.cn). The Clinical Trial Center Office of Eye Hospital and School of Ophthalmology and Optometry of Wenzhou Medical University reviewed the entire study. None of the authors have any conflicting interests to disclose.

Availability of data and materials The ethical approval does not permit to sharing the entire data that we have acquired, but the datasets used and analysed in our study are available from the corresponding author.

\section{References}

1. Govindan M, Mohney BG, DiehI NN, Burke JP. Incidence and types of childhood exotropia: a population-based study. Ophthalmology. 2005;112(1):104-8. https://doi.org/10.1016/j.ophtha.2004.07.033.

2. Mohney BG. Common forms of childhood strabismus in an incidence cohort. Am J Ophthalmol. 2007;144(3):465. 7.https://doi.org/10.1016/j.ajo.2007.06.011.

3. Chen X, Fu Z, Yu J, Ding H, Bai J, Chen J, et al. Prevalence of amblyopia and strabismus in Eastern China: results from screening of preschool children aged 36-72 months. $\mathrm{Br} \mathrm{J}$ Ophthalmol. 2016;100(4):515-9. .https://doi.org/10.1136/bjophthalmol-2015-306999. 
4. Yamada T, Hatt SR, Leske DA, Holmes JM. Health-related quality of life in parents of children with intermittent exotropia. J AAPOS. 2011;15(2):135-9. https://doi.org/10.1016/j.jaapos.2010.11.020.

5. Wang Y, Xu M, Yu H, Xu J, Hou F, Zhou J, et al. Health-related quality of life correlated with the clinical severity of intermittent exotropia in children. Eye (Lond). 2020;34(2):400-7. .https://doi.org/10.1038/s41433-019-0557-1.

6. Yu X, Ji Z, Yu H, Xu M, Xu J. Exotropia Is the Main Pattern of Childhood Strabismus Surgery in the South of China: A Six-Year Clinical Review. J Ophthalmol. 2016. 2016:1489537.https://doi.org/10.1155/2016/1489537.

7. Holmes JM, Hatt SR, Leske DA. Is intermittent exotropia a curable condition? Eye (Lond). 2015;29(2):171-6. https://doi.org/10.1038/eye.2014.268.

8. Pineles SL, Ela-Dalman N, Zvansky AG, Yu F, Rosenbaum AL. Long-term results of the surgical management of intermittent exotropia. J AAPOS. 2010;14(4):298-304. https://doi.org/10.1016/j.jaapos.2010.06.007.

9. Yang M, Chen J, Shen T, Kang Y, Deng D, Lin X, et al. Clinical Characteristics and Surgical Outcomes in Patients With Intermittent Exotropia: A Large Sample Study in South China. Med (Baltim). 2016;95(5):e2590. https://doi.org/10.1097/MD.0000000000002590.

10. Liu Y, Liu C, Zhang W, Chen X, Zhao K. Model of a Support Vector Machine to Assess the Functional Cure for Surgery of Intermittent Exotropia. Sci Rep. 2019;9(1):8321. https://doi.org/10.1038/s41598019-38969-x.

11. Chougule P, Kekunnaya R. Surgical management of intermittent exotropia: do we have an answer for all? BMJ Open Ophthalmol. 2019;4(1):e000243. https://doi.org/10.1136/bmjophth-2018-000243.

12. Park H, Kim WJ, Kim MM. The Stabilization of Postoperative Exo-drift in Intermittent Exotropia after Surgical Treatment. Korean J Ophthalmol. 2016;30(1):60 - 5. https://doi.org/10.3341/kjo.2016.30.1.60.

13. Kim WJ, Kim MM. The clinical course of recurrent intermittent exotropia following one or two surgeries over 24 months postoperatively. Eye (Lond). 2014;28(7):819 - 24. https://doi.org/10.1038/eye.2014.93.

14. Jeoung JW, Lee MJ, Hwang JM. Bilateral lateral rectus recession versus unilateral recess-resect procedure for exotropia with a dominant eye. Am J Ophthalmol. 2006;141(4):683-8. .https://doi.org/10.1016/j.ajo.2005.11.021.

15. Koklanis K, Georgievski Z. Recurrence of intermittent exotropia: factors associated with surgical outcomes. Strabismus. 2009;17(1):37-40. https://doi.org/10.1080/09273970802678750.

16. Chryssanthou G. Orthoptic Management of Intermittent Exotropia. Am Orthopt J. 2018;24(1):69-72. .https://doi.org/10.1080/0065955x.1974.11982349.

17. Singh V, Roy S, Sinha S. Role of orthoptic treatment in the management of intermittent exotropia. Indian J Ophthalmol. 1992;40(3):83-5.

18. France LW. The Role of Orthoptic Therapy in Exodeviations: A Basis for Orthoptic Referral. Am Orthopt J. 1992;42(1):52-64. https://doi.org/10.1080/0065955X.1992.11981895. 
19. Coffey B, Wick B, Cotter S, Scharre J, Horner D. Treatment options in intermittent exotropia: a critical appraisal. Optom Vis Sci. 1992;69(5):386-404. https://doi.org/10.1097/00006324-19920500000008.

20. Figueira EC, Hing S. Intermittent exotropia: comparison of treatments. Clin Exp Ophthalmol. 2006;34(3):245 - 51.https://doi.org/10.1111/j.1442-9071.2006.01199.x.

21. Investigator PEyeD, Writing G, Donahue C, Chandler SP, Holmes DL, Arthur JM. BW, et al. A Randomized Trial Comparing Bilateral Lateral Rectus Recession versus Unilateral Recess and Resect for Basic-Type Intermittent Exotropia. Ophthalmology. 2019;126(2):305 - 17. https://doi.org/10.1016/j.ophtha.2018.08.034.

22. Chan AW, Tetzlaff JM, Gotzsche PC, Altman DG, Mann H, Berlin JA, et al. SPIRIT 2013 explanation and elaboration: guidance for protocols of clinical trials. BMJ. 2013;346:e7586. .https://doi.org/10.1136/bmj.e7586.

23. Hatt SR, Leske DA, Yamada T, Bradley EA, Cole SR, Holmes JM. Development and initial validation of quality-of-life questionnaires for intermittent exotropia. Ophthalmology. 2010;117(1):163-8. e1.https://doi.org/10.1016/j.ophtha.2009.06.038.

24. Mohney BG, Holmes JM. An office-based scale for assessing control in intermittent exotropia. Strabismus. 2006;14(3):147-50. https://doi.org/10.1080/09273970600894716.

25. Wang L, Wu Q, Kong X, Li Z. Comparison of bilateral lateral rectus recession and unilateral recession resection for basic type intermittent exotropia in children. Br J Ophthalmol. 2013;97(7):870-3. .https://doi.org/10.1136/bjophthalmol-2013-303167.

26. Xie F, Zhao K, Zhang W. Comparison of surgical outcomes between bilateral recession and unilateral recession-resection in moderate-angle intermittent exotropia. J AAPOS. 2019;23(2):79 e1e7.https://doi.org/10.1016/j.jaapos.2018.11.007.

\section{Tables}

Table 1. Inclusion and exclusion criteria 
Inclusion criteria

1. Patient is 7-17 years old.

2. Amount of exodeviation measured using PACT at 1 month after IXT surgery meets any of the following:

2.1 Exodeviation $\otimes 10 P D$ at near and distance.

2.2 Ortho at near, and exodeviation $\otimes 10 P D$ at distance.

2.3 Ortho at distance, and exodeviation $\triangle 10 \mathrm{PD}$ at near.

3. Best corrected visual acuity (BCVA) in the worse eye is 0.1 logMAR or better and there has been no previous diagnosis or treatment for amblyopia.

4. Participant wore spectacles for at least 1 week if refractive error meets any of the following:

4.1 Myopia囚-0.50 D and >-6.00 D SE in either eye.

4.2 Anisometropia $\otimes 1.50 \mathrm{D}$ SER.

4.3 No hyperopia of more than +3.50 D SER in either eye.

5. Refractive correction for participants meeting the above refractive error criteria must meet the following guidelines:

5.1 For myopia, the intent is to fully correct.

5.2 For hyperopia, the spherical component can be reduced at investigator discretion as the principal of maximum plus to maximum visual acuity.

5.3 For astigmatism, cylinder must be within $0.25 \mathrm{D}$ of full correction and axis must be within 5 degrees of full correction.

5.4 Myopic participants should not take any intervention for myopia control, such as orthokeratology, peripheral defocus lenses, low-concentrate atropine.

6. No atropine was used within the previous month.

7. Patient had gestational age $>34 \mathrm{wks}$ and birth weight $>1500 \mathrm{~g}$.

8. Parents and participant understand protocol and are willing to accept randomization to binocular training group or sham-control group.

9. Patient had not received prior vision training or orthoptics for any reason.

10. The location of the home address is not very far from the hospital, and if assigned to the training group, training 1-2 times a week at hospital is acceptable.

Exclusion criteria

1. Coexisting vertical deviation $₫ 5 \mathrm{PD}$.

2. Esodeviation $₫ 5 \mathrm{PD}$ at near or distance 
3. Complaints of diplopia 1 month after surgery in the primary and reading positions.

4. Limitation of ocular rotations resulting from restrictive or paretic strabismus.

5. Craniofacial malformations affecting the orbits.

6. Interocular visual acuity difference more than $0.2 \log M A R$.

7. High $\mathrm{AC} / \mathrm{A}$ ratio (exclude $>6: 1$ by gradient method).

8. Prior strabismus surgery or botulinum toxin injection.

9. Prior intraocular or refractive surgery.

10. Significant neurological impairment such as cerebral palsy. Participants with mild speech or learning disabilities or both are eligible.

PACT: simultaneous prism and alternate cover test; IXT: intermittent exotropia; PD: prism diopter; SE: spherical equivalent refraction; $\mathrm{AC} / \mathrm{A}$ : accommodative convergence/accommodation 
Table 2

Hospital based and home-based orthoptic therapy procedure

\begin{tabular}{|c|c|c|c|}
\hline Phase & $\begin{array}{l}\text { Therapy } \\
\text { location }\end{array}$ & Training technique & Endpoint \\
\hline \multirow[t]{8}{*}{$\begin{array}{l}\text { Phase } \\
1\end{array}$} & $\begin{array}{l}\text { Hospital- } \\
\text { based } \\
\text { therapy }\end{array}$ & $\begin{array}{l}\text { Training with major } \\
\text { amblyoscope to build } \\
\text { fine retina } \\
\text { correspondence }\end{array}$ & Achieve simultaneous vision and build fusion \\
\hline & & $\begin{array}{l}\text { Cheiroscopic drawing } \\
\text { with single oblique } \\
\text { stereoscope }\end{array}$ & Achieve simultaneous vision and build fusion \\
\hline & & $\begin{array}{l}\text { Vectograms (quoits } \\
\text { or clowns) for } \\
\text { convergence }\end{array}$ & $33 \mathrm{BO}$ for twice for patients without stereopsis \\
\hline & & $\begin{array}{l}\text { Increasing fusional } \\
\text { convergence based } \\
\text { on stereoscopic } \\
\text { stimuli }\end{array}$ & $34 \mathrm{BO}$ for twice for patient with stereopsis \\
\hline & $\begin{array}{l}\text { Home } \\
\text { therapy }\end{array}$ & Red/green bar reader & Maintain single binocular without suppression \\
\hline & & Barrel card & Easily fuse three dots \\
\hline & & Brock string & Successfully converge to $2.5 \mathrm{~cm}$ from his or her nose. \\
\hline & & $\begin{array}{l}\text { Letter chart } \\
\text { monocular } \\
\text { accommodative } \\
\text { facility (MAF) }\end{array}$ & $\begin{array}{l}\text { Start from } 20 / 50 \text {, go to } 20 / 40 \text { or } 20 / 30 \text {, when result of } \\
\text { MAF is } 17.5 \mathrm{cpm}\end{array}$ \\
\hline \multirow[t]{5}{*}{$\begin{array}{l}\text { Phase } \\
2\end{array}$} & $\begin{array}{l}\text { Hospital- } \\
\text { based } \\
\text { vision } \\
\text { therapy }\end{array}$ & $\begin{array}{l}\text { Training with major } \\
\text { amblyoscope to } \\
\text { increase fusional } \\
\text { convergence }\end{array}$ & $\begin{array}{l}\text { Fusional convergence reserve is more than }+25^{\circ} \\
\text { (based on different subjective angle of exodeviation, } \\
\text { the amount of fusional convergence demand is } \\
\text { different) }\end{array}$ \\
\hline & & $\begin{array}{l}\text { Vectograms (quoits } \\
\text { or clowns) for } \\
\text { divergence }\end{array}$ & $15 \mathrm{BI}$ with stereopsis \\
\hline & & $\begin{array}{l}\text { Aperture rule for } \\
\text { convergence and } \\
\text { divergence }\end{array}$ & 12 pictures for convergence, 7 pictures for divergence. \\
\hline & & $\begin{array}{l}\text { Random vergence } \\
\text { facility based on } \\
\text { stereoscopic stimuli }\end{array}$ & More than 650 times in 15 minutes \\
\hline & $\begin{array}{l}\text { Home- } \\
\text { based } \\
\text { vision } \\
\text { therapy }\end{array}$ & Aperture rule & 7 for divergence and 12 for convergence \\
\hline
\end{tabular}




\begin{tabular}{|c|c|c|c|}
\hline Phase & $\begin{array}{l}\text { Therapy } \\
\text { location }\end{array}$ & Training technique & Endpoint \\
\hline & & Letter chart BAF & More than $17.5 \mathrm{cpm}(20 / 30)$ \\
\hline & & $\begin{array}{l}\text { Computerized } \\
\text { binocular vision } \\
\text { therapy procedure }\end{array}$ & $\begin{array}{l}\text { Achieve clear single binocular vision with } 40 \text { to } 50 \mathrm{BO} \\
\text { and } 10 \text { to } 15 \mathrm{BI} \text {. }\end{array}$ \\
\hline \multirow[t]{8}{*}{$\begin{array}{l}\text { Phase } \\
3\end{array}$} & $\begin{array}{l}\text { Hospital- } \\
\text { based } \\
\text { vision } \\
\text { therapy }\end{array}$ & $\begin{array}{l}\text { Training with major } \\
\text { amblyoscope to } \\
\text { increase fusional } \\
\text { convergence }\end{array}$ & $\begin{array}{l}\text { Fusional convergence reserve is more than }+25^{\circ} \\
\text { (based on different subjective angle of strabismus, } \\
\text { amount of fusional convergence demand is different) }\end{array}$ \\
\hline & & $\begin{array}{l}\text { Aperture rule with } \\
\text { lens or prism flipper }\end{array}$ & Accomplish with \pm 2.00 lens flipper \\
\hline & & $\begin{array}{l}\text { Random vergence } \\
\text { facility based on } \\
\text { stereoscopic stimuli }\end{array}$ & More than 650 times in 15 minutes \\
\hline & & $\begin{array}{l}\text { Bernell-O-Scope for } \\
\text { vergence facility }\end{array}$ & 8 pictures for $\mathrm{BI}$ and $\mathrm{BO}$ each \\
\hline & & $\begin{array}{l}\text { Free space fusion } \\
\text { with lifesaver card }\end{array}$ & Easily accomplish convergence and divergence \\
\hline & & $\begin{array}{l}\text { VR training that } \\
\text { combines pursuit and } \\
\text { saccade }\end{array}$ & Accuracy ratio more than $90 \%$ in increasing difficulty \\
\hline & $\begin{array}{l}\text { Home- } \\
\text { based } \\
\text { vision } \\
\text { therapy }\end{array}$ & $\begin{array}{l}\text { Aperture rule with } \pm \\
2.00 \text { lens flipper }\end{array}$ & Accomplish 7 in divergence and 12 in convergence \\
\hline & & Lifesaver card & Easily accomplish divergence and convergence \\
\hline
\end{tabular}

\section{Figures}




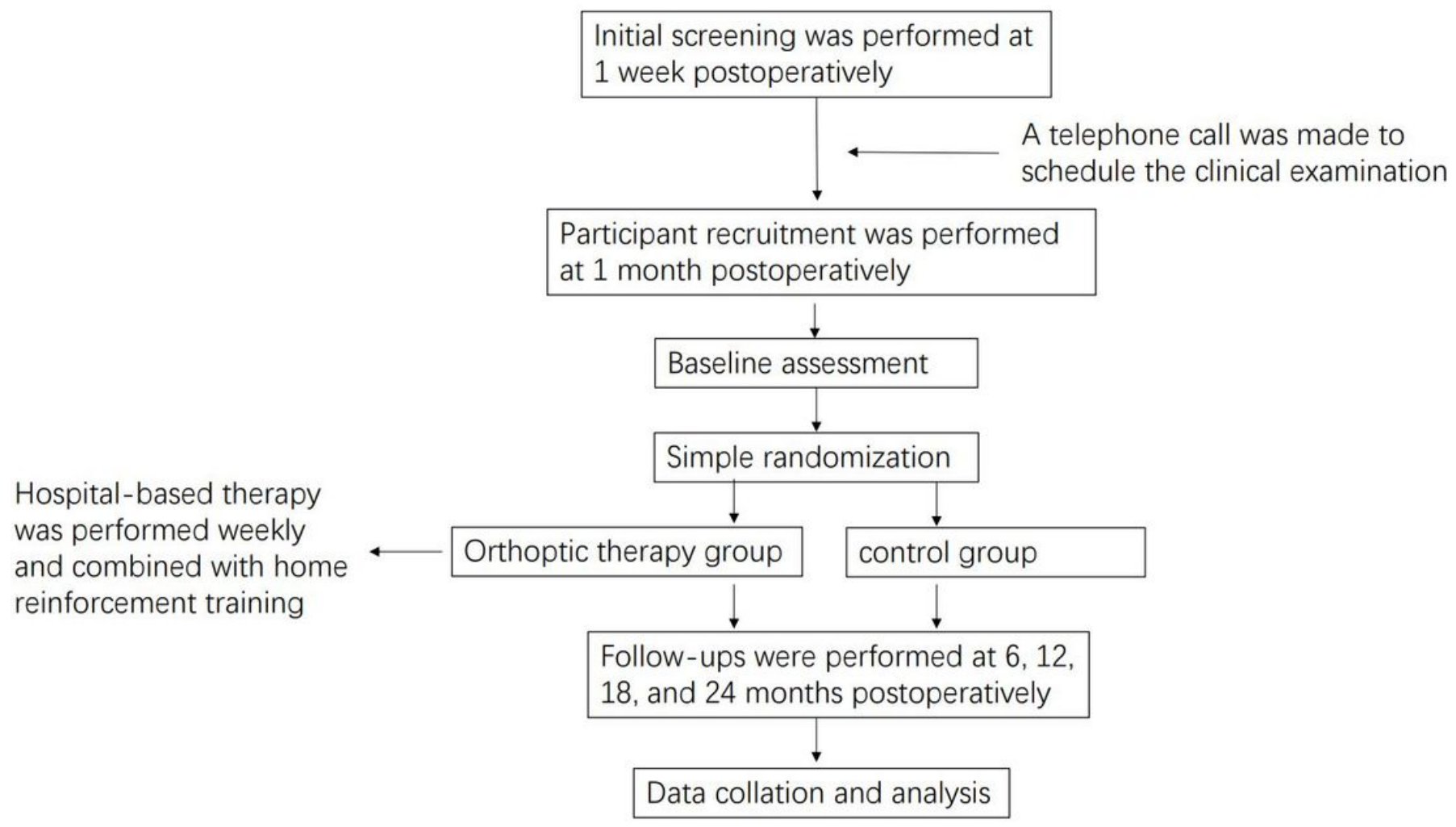

Figure 1

Flowchart of our current study.

\section{Supplementary Files}

This is a list of supplementary files associated with this preprint. Click to download.

- SPIRITchecklist.docx

- SPRITFIGURE.pdf 